Pacific Journal of Mathematics

ANALYSIS OF INVARIANT MEASURES IN DYNAMICAL 


\title{
ANALYSIS OF INVARIANT MEASURES IN DYNAMICAL SYSTEMS BY HAUSDORFF MEASURE
}

\author{
WM. DOUglas Withers
}

\begin{abstract}
Hausdorff measure is a preliminary concept in the definition of Hausdorff dimension, which is one concept of the degree of singularity of a finite measure. In general, Hausdorff measure does not permit as detailed an analysis of an arbitrary natural invariant measure arising from a dynamical system as Lebesgue measure permits of an absolutely continuous measure. It is shown that even for a dynamical system as simple as a modified baker's transformation, the natural invariant measure has no representation as an indefinite integral with respect to any Hausdorff measure. However, Hausdorff measure can be used to compare different natural invariant measures according to degree of singularity even when their Hausdorff dimensions are identical.
\end{abstract}

1. Introduction. In this article we seek to illustrate some of the capabilities and limitations of Hausdorff measure for the analysis of invariant measures in dynamical systems in more detail than is possible with Hausdorff dimension alone.

Hausdorff dimension is a concept of the size of a set or the degree of singularity of a measure. In recent years it has often been used for the study of dynamical systems because many of the sets of interest which arise are of Lebesgue measure zero and many of the measures of interest whch arise are singular with respect to Lebesgue measure. Thus Lebesgue measure is of little or no aid in the analysis of these sets and measures. The Hausdorff dimension of a set of Lebesgue measure zero or a measure singular with respect to Lebesgue measure can, however, range over a wide spectrum of possible values; such sets and measures can thus be distinguished and classified by their Hausdorff dimensions.

Hausdorff measure is a necessary preliminary to the definition of Hausdorff dimension and in cases where the Hausdorff dimension fails to distinguish between two sets or between two measures (their Hausdorff dimensions being the same) it is sometimes possible to compare their sizes using Hausdorff measure. 
Perhaps the greatest possible amount of information concerning a measure $\mu$ is given by a representation

$$
\mu E=\int_{E} \delta(x) d \Lambda(x),
$$

where $\delta$ is a density function and $\Lambda$ is a "reference measure" which is uniform at each point, such as Hausdorff measure is. In several cases, an invariant measure arising from a dynamical system does have such a representation (we give some examples in \$3) and in view of the natural relationship between the Hausdorff dimension of an invariant measure in a system and other properties of the system (for example, see [12]) and the relationship of Hausdorff measure to Hausdorff dimension, it seems reasonable to suppose that the invariant measure arising from a dynamical system might always have such a representation.

In this article we present a simple example of a dynamical system, namely, a modified baker's transformation, which shows that such is not the case. A by-product of the arguments used to establish this result is an illustration of the use of Hausdorff measure to provide a finer classification of measures than is possible by the use of Hausdorff dimension alone.

In $\S 2$ we review the definitions of Hausdorff measure and Hausdorff dimension. In $\$ 3$ we give some examples of dynamical systems giving rise to invariant measures having representations as integrals of Hausdorff measure. In $\$ 4$ we present our example of a system giving rise to an invariant measure without such a representation. In $\$ 5$ we use Hausdorff measure to classify a family of measures akin to the example of $\S 4$ which have the same Hausdorff dimension and we discuss the extension of our methods to general invariant measures in dynamical systems.

2. Definition of Hausdorff measure and Hausdorff dimension. Let $\Omega$ be a metric space. Let $\lambda$ be a function from some $(0, \eta)$ to $(0, \infty)$. The function $\lambda$ need not be continuous or increasing. For a subset $E$ of $\Omega$ we define the Hausdorff measure of $E$ (associated with $\lambda$ ), denoted $m_{\lambda} E$, by

$$
m_{\lambda} E=\sup _{0<\varepsilon<\eta} \inf _{\left\{A_{n}\right\}} \sum \lambda\left(\operatorname{diam} A_{n}\right),
$$

where $\left\{A_{n}\right\}$ ranges over all coverings of $E$ by open balls in $\Omega$ with $\operatorname{diam} A_{n}<\varepsilon$. Note that different choices of $\lambda$ and different metrics on $\Omega$ can yield different measures $m_{\lambda}$. We call $\lambda$ an index function for $m_{\lambda}$. The $m_{\lambda}$-measurable sets in $\Omega$ include all Borel sets. Many familiar measures can be obtained as special cases of Hausdorff measure. For example, if 
$\Omega=\mathbf{R}^{n}$ and $\lambda(t)=t^{n}$, then $m_{\lambda}$ is just a constant times $n$-dimensional Lebesgue measure. If we set $\lambda \equiv 1$, then $m_{\lambda}$ is just counting measure; that is, $m_{\lambda}\{x\}=1$ for each $x$ in $\Omega$.

If $\Omega$ is a linear space, and $\lambda(t)=t^{d}$ for some $d$, then the measure $m_{\lambda}$ has an interesting scaling property. Let $E$ be a set in $\Omega$ and $a$ be a real positive number. Then

$$
m_{\lambda}(a E)=a^{d} m_{\lambda} E
$$

where $a E=\{a x: x \in E\}$ is the set $E$ magnified by the factor $a$. Compare this to the scaling property of $n$-dimensional Lebesgue measure. Also, $m_{\lambda}$ is translation-invariant.

For the definition of the Hausdorff dimension of a set, we set $\lambda(t)=t^{d}$ for some unspecified value of $d$. Then $m_{\lambda} E$ is a function of $d$ as well as $E$. If we fix $E$ and vary $d$, we find that there is a critical value $d_{0}$ for $d$ such that $m_{\lambda} E=\infty$ when $d<d_{0}$ and $m_{\lambda} E=0$ when $d>d_{0}$. The number $d_{0}$ is the Hausdorff dimension of the set $E$, which we denote $\operatorname{dim} E$.

The Hausdorff dimension of a finite measure $\mu$ on $\Omega$ can be defined in terms of the Hausdorff dimension of subsets of $\Omega$. Let

$$
d_{0}=\inf \{\operatorname{dim} E: \mu E>0\} ;
$$

then $d_{0}$ is the Hausdorff dimension of $\mu$, denoted $\operatorname{dim} \mu$. Alternatively, $\operatorname{dim} \mu$ is the unique number such that, letting $\lambda(t)=t^{d}, \mu$ is absolutely continuous with respect to $m_{\lambda}$ when $d<\operatorname{dim} \mu$ and $\mu$ is singular with respect to $m_{\lambda}$ when $d>\operatorname{dim} \mu$.

For a set $E$ and a Hausdorff measure $m_{\lambda}, m_{\lambda} E$ may be zero, or positive, or positive and finite. These are analogous to the range of possibilities for measures and, for a finite measure $\mu$, correspond to $\mu$ being singular with respect to $m_{\lambda}$, absolutely continuous with respect to $m_{\lambda}$, or having a representation as an integral of $m_{\lambda}$, respectively.

Let $\mu$ and $\nu$ be finite measures. If $\operatorname{dim} \mu<\operatorname{dim} \nu$, then there exists a Hausdorff measure $m_{\lambda}$ such that $\mu$ is singular with respect to $m_{\lambda}$ while $\nu$ is absolutely continuous with respect to $m_{\lambda}$; for example, we may take $\lambda(t)=t^{d}$ with $d=(\operatorname{dim} \mu+\operatorname{dim} \nu) / 2$. Thus we think of $\mu$ as being "more singular" than $\nu$. Even in some cases where $\operatorname{dim} \mu=\operatorname{dim} \nu$, there exists $m_{\lambda}$ such that $\mu$ is singular with respect to $m_{\lambda}$ while $\nu$ is absolutely continuous with respect to $m_{\lambda}$; an example of this situation is given in $\S 5$. In this case, we could distinguish $\mu$ as being more singular than $\nu$ even though $\operatorname{dim} \mu=\operatorname{dim} \nu$. This is what was referred to in the introduction as Hausdorff measure providing a finer classification of measures than Hausdorff dimension alone. For a detailed treatment of this sort of classification of measures, see Rogers and Taylor [8]. 
3. Examples of invariant measures representable by integrals of Hausdorff measure.

\section{A. The logistic map.}

Let $f:[0,1] \rightarrow[0,1]$ be given by $f(x)=4 x(1-x)$. Let us define a sequence $\mu_{n}$ of measures on $[0,1]$ by setting $\mu_{0}$ equal to Lebesgue measure on $[0,1]$ and defining $\mu_{n+1} E=\mu_{n} f^{-1}(E)$. The sequence $\mu_{n}$ converges weakly to a limit measure $\mu$, which is the invariant measure for the system. Given an initial point $x_{0}$ chosen uniformly at random from $[0,1]$ and letting $x_{n+1}=f\left(x_{n}\right), \mu$ is the probability distribution of $x_{n}$ for $n$ very large.

The measure $\mu$ has the integral representation

$$
\mu E=\int_{E} \frac{d m(x)}{\pi \sqrt{x(1-x)}},
$$

the notation $d m(x)$ denoting integration with respect to Lebesgue measure, which is a special case of Hausdorff measure. This representation gives a much better picture of $\mu$ than the bare fact that $\operatorname{dim} \mu=1$; we can see, for example, that the density of $\mu$ goes to infinity at 0 and 1 .

The logistic map may be the most widely-known example of a dynamical system; for an introduction to its theory see Collet and Eckmann [2]. The more general map $f_{a}(x)=a x(1-x)$ is more difficult to analyse, but in the work of Jakobson [4] and Benedicks and Carleson [1] it is shown that for a set of values of the parameter $a$ of positive measure, the map $f_{a}$ generates an invariant measure which is absolutely continuous and thus can be represented as an integral of Lebesgue measure.

B. For complex $z$, let $f(z)=z^{2}-2 \bar{z}$. This map has as a chaotic attractor the region of $\mathbf{C}$ where

$$
\rho(z)=4\left(z^{3}+\bar{z}^{3}\right)-(z \bar{z})^{2}-18 z \bar{z}+27 \geq 0 .
$$

Moreover, the invariant measure $\mu$ generated by $f$ is absolutely continuous and has the integral representation:

$$
\mu E=\int_{E} \frac{3 d m_{2}(z)}{\pi^{2} \sqrt{\rho(z)}},
$$

where $d m_{2}(z)$ denotes integration with respect to Lebesgue area measure in C. Again, this representation tells us, for example, that the density goes to infinity on the boundary of the attractor and that the measure is invariant under multiplication of $z$ by $-1 / 2+i \sqrt{3} / 2$. 
This example and the mapping $f(x)=4 x(1-x)$ above are members of a class of mappings with many special properties, among which is the existence of an absolutely continuous invariant measure with an algebraic density function. A more complete description is given in Withers [11].

C. A modified baker's transformation.

Let $S$ be the square $[0,1] \times[0,1]$, endowed with the square metric, so that $\operatorname{dist}\left(\left(x_{1}, y_{1}\right),\left(x_{2}, y_{2}\right)\right)=\sup \left\{\left|x_{2}-x_{1}\right|,\left|y_{2}-y_{1}\right|\right\}$. We define $f: S \rightarrow$ $S$ by

$$
f(x, y)= \begin{cases}(x / 3,2 y) & \text { if } y<1 / 2, \\ ((x+2) / 3,2 y-1) & \text { if } y \geq 1 / 2 .\end{cases}
$$

Note $f(S) \neq S$. As before we define a sequence $\mu_{n}$ of measures on $S$ by setting $\mu_{0}$ equal to Lebesgue area measure on $S$ and letting $\mu_{n+1} E=$ $\mu_{n} f^{-1}(E)$. Then the sequence $\mu_{n}$ converges weakly to a limit measure $\mu$ on $S$. The measure $\mu$ is supported on the set $C \times[0,1]$, where $C$ is the canonical Cantor set $(C=[0,1]-(1 / 3,2 / 3)-(1 / 9,2 / 9)-(7 / 9,8 / 9)$ - ...), which has zero area. Thus $\mu$ cannot be represented as an integral of Lebesgue measure.

However, let us set $\lambda(t)=t^{d}$, where $d=\log _{3} 6=\operatorname{dim} \mu$. Then $\mu$ has the representation

$$
\mu E=\int_{E} \delta(x, y) d m_{\lambda}(x, y),
$$

where $\delta(x, y)=1$ if $x \in C, \delta(x, y)=0$ otherwise, and the notation $d m_{\lambda}(x, y)$ denotes integration with respect to the measure $m_{\lambda}$. Again, from this representation we can see, for example, that $\mu$ is of uniform density on the set $C \times[0,1]$ and of zero density outside this set, which cannot be inferred just from the Hausdorff dimension of $\mu$. A generalization of this example is the subject of the next section.

D. Let $M$ be a Riemann manifold and $f: M \rightarrow M$ a $C^{1+\varepsilon}$ map which is conformal; i.e., its derivative is a scalar times an isometry. Let $J$ be a compact subset of $M$ with an open neighborhood $V$ satisfying the following conditions:

(i) There exist $C>0$ and $\alpha>1$ such that $\left\|\left(f^{n}\right)^{\prime}\right\| \geq C \alpha^{n}$ for all $x \in J$ and $n \geq 1$.

(ii) $J=\left\{x \in V: f^{n}(x) \in V\right.$ for all $\left.n>0\right\}$.

(iii) For every nonempty open set $U$ intersecting $J$ there exists $n>0$ such that $J \subset f^{n}(U)$. 
These conditions make $J$ a repeller for $f$ and imply in particular that $J$ is invariant under $f$. For a function $\psi: J \rightarrow \mathbf{R}$, we define the pressure $p(\psi)$ by

$$
p(\psi)=\sup \left\{h(\mu)+\int \psi d \mu\right\},
$$

where $\mu$ ranges over all probability measures on $J$ invariant under $f$ and $h$ is the entropy of $\mu$ with respect to $f$. Let $\varphi: J \rightarrow \mathbf{R}$ be Hölder continuous. There is then a unique Radon measure $\mu$ on $J$ such that $p(\varphi+\psi)-p(\varphi) \geq \int \psi d \mu$ for all $\psi$. The measure $\mu$ is a probability measure invariant under $f$ and is called the Gibbs measure associated with $\varphi$.

In the case where $\varphi(x)=-\operatorname{dim} J \log \left\|f^{\prime}\right\|$, it follows from a theorem of Ruelle [9] that $\mu$ has a representation as an integral:

$$
\mu E=\int_{E} \delta(x) d m_{\lambda}(x)
$$

where $\lambda(t)=t^{\operatorname{dim} J}$.

4. The modified baker's transformation. Our system has three parameters: $p, q$, and $r$, all positive numbers such that $p<1$ and $q+r<1$. Let us define $g_{0}, g_{1}:[0,1] \rightarrow[0,1]$ by $g_{0}(x)=q x, g_{1}(x)=r x$ $+1-r$. Let $S$ be the square $[0,1] \times[0,1]$. We define $f: S \rightarrow S$ by

$$
f(x, y)= \begin{cases}\left(g_{0}(x), y / p\right) & \text { if } y \leq p, \\ \left(g_{1}(x),(y-p) /(1-p)\right) & \text { if } y>p .\end{cases}
$$

As in example $\mathrm{C}$ of the previous section, $f$ generates an invariant probability measure $\mu$ on $S$. It can be shown that the measure $\mu$ can be written as a product measure $\nu \times m$, where $\nu$ is a finite measure on the $x$-axis given by $\nu E=\mu(E \times[0,1])$ and $m$ is Lebesgue measure on the $y$-axis. It can be further shown that $\mu$ has an integral representation as an integral of Hausdorff measure if and only if $\nu$ does. We can thus simplify our problem by considering the measure $\nu$ on $[0,1]$; we ask whether there exist an index function $\lambda$ and a density $\delta$ such that

$$
\nu E=\int_{E} \delta(x) d m_{\lambda}(x) .
$$

Our computations will be simplified if we restrict consideration to the following set $\Xi$ of intervals. Each interval is indexed by indices $\left(\gamma_{1}, \gamma_{2}, \ldots, \gamma_{n}\right)$, each $\gamma_{i} \in\{0,1\}$. We define

$$
I\left(\gamma_{1}, \gamma_{2}, \ldots, \gamma_{n}\right)=g_{\gamma_{1}}\left(g_{\gamma_{2}}\left(\cdots g_{\gamma_{n}}([0,1]) \cdots\right)\right) \text {. }
$$


We call the number $n$ of indices for $J=I\left(\gamma_{1}, \gamma_{2}, \ldots, \gamma_{n}\right)$ the degree of $J$ and denote it $\operatorname{deg} J$. Note that with $q+r<1$, if $\operatorname{deg} J=\operatorname{deg} K$ and $J \neq K$, then $J \cap K=\varnothing$. Let us define

$$
F_{n}=\bigcup\{J \in \Xi: \operatorname{deg} J=n\} \text {. }
$$

Note $\nu\left([0,1]-F_{n}\right)=0$. We further define

$$
F=\bigcap_{n=0}^{\infty} F_{n}
$$

then $\nu([0,1]-F)=0$ and we can ignore this part of the interval when seeking an integral representation. We also introduce a modification of Hausdorff measure.

4.1. Definition. Let $\lambda:(0, \eta) \rightarrow(0, \infty)$. Let $E \subset F$. We define the modified Hausdorff measure of $E$ (associated with $\lambda$ ), denoted $M_{\lambda} E$, by

$$
M_{\lambda} E=\sup _{0<\varepsilon<\eta} \inf _{\left\{I_{n}\right\}} \sum \lambda\left(\operatorname{diam} I_{n}\right),
$$

where $\left\{I_{n}\right\}$ ranges over all coverings of $E$ by elements of $\Xi$ with $\operatorname{diam} I_{n}<\varepsilon$.

The only modification introduced is that the covering of $E$ must be contained in the set $\Xi$.

The measure $\mu$ can be represented as an integral with respect to a modified Hausdorff measure $M_{\lambda}$ if and only if it can be represented as an integral with respect to the ordinary Hausdorff measure $m_{\lambda}$. This follows from the next lemma, of which the proof is straightforward.

4.2. Lemma. Suppose $\lambda$ is such that for each $a>0, \lambda(a t) / \lambda(t)$ is bounded as $t$ goes to 0 . (On the real line, any Hausdorff measure of interest can be obtained from such an index function.) Let $\alpha$ be such that

$$
\lambda(t)<\alpha\left(\lambda\left(\frac{q t}{1-q-r}\right)+\lambda\left(\frac{r t}{1-q-r}\right)\right)
$$

for $t$ sufficiently small. Then for each $E \subset F$,

$$
m_{\lambda} E \leq M_{\lambda} E \leq \alpha m_{\lambda} E \text {. }
$$

Our main tool for seeking a representation for $\mu$ as an integral of modified Hausdorff measure $M_{\lambda}$ is the following theorem. 
4.3. ThEOREM. For $\lambda:(0, \eta) \rightarrow(0, \infty)$ and a finite measure $\nu$ on $F$, we define a function $D^{\lambda} \nu:[0,1] \rightarrow(0, \infty)$ as follows:

$$
D^{\lambda} \nu(x)=\inf _{0<\varepsilon<\eta} \sup _{x \in I \in \Xi} \frac{\nu I}{\lambda(\operatorname{diam} I)},
$$

where $\operatorname{diam} I<\varepsilon$. Let $T_{0}=\left\{x: D^{\lambda} \nu(x)=0\right\}, T_{+}=\left\{x: 0<D^{\lambda} \nu(x)<\right.$ $\infty\}$, and $T_{\infty}=\left\{x: D^{\lambda} \nu(x)=\infty\right\}$. Then:

(i) $\nu$ is absolutely continuous with respect to $M_{\lambda}$ if and only if $\nu T_{\infty}=0$.

(ii) $\nu$ is singular with respect to $M_{\lambda}$ if and only if $\nu T_{0}=\nu T_{+}=0$.

(iii) $\nu$ has a representation as an integral with respect to $M_{\lambda}$ if and only if $\nu T_{0}=\nu T_{\infty}=0$.

Proof. Rogers [7, Theorem 67] is exactly like this except using standard Hausdorff measure $m_{\lambda}$ rather than modified Hausdorff measure $M_{\lambda}$. The proof of this theorem is a straightforward adaptation of his arguments.

We now make a symbolic analogy which is frequently used in the study of dynamical systems. Let us consider in more detail the properties of the intervals $I\left(\gamma_{1}, \gamma_{2}, \ldots, \gamma_{n}\right)$. Note first that if $k \leq n$ then $I\left(\gamma_{1}, \gamma_{2}, \ldots, \gamma_{n}\right) \subset I\left(\beta_{1}, \beta_{2}, \ldots, \beta_{k}\right)$ if and only if $\gamma_{i}=\beta_{i}$ for $1 \leq i \leq k$. From the definition of $I\left(\gamma_{1}, \gamma_{2}, \ldots, \gamma_{n}\right)$ we see that

$$
\operatorname{diam} I\left(\gamma_{1}, \gamma_{2}, \ldots, \gamma_{n}\right)=s_{\gamma_{1}} s_{\gamma_{2}} \cdots s_{\gamma_{n}},
$$

where $s_{0}=q, s_{1}=r$. Using the fact that $\mu$ is uniform in the $y$-direction and invariant under $f$, we have that $\nu g_{0}(E)=p \nu E$ and $\nu g_{1}(E)=$ $(1-p) \nu E$ for any $\nu$-measurable set $E$. It follows that

$$
\nu I\left(\gamma_{1}, \gamma_{2}, \ldots, \gamma_{n}\right)=t_{\gamma_{1}} t_{\gamma_{2}} \cdots t_{\gamma_{n}},
$$

where $t_{0}=p, t_{1}=1-p$.

Now we consider $D^{\lambda} \nu(x)$. Recall that $\nu$ has a representation as an integral of the Hausdorff measure $m_{\lambda}$ if and only if the set of points where $D^{\lambda} \nu(x)=0$ or $D^{\lambda} \nu(x)=\infty$ is a $\nu$-null set. We restate this in probabilistic terms. Let $x$ be chosen at random, distributed according to $\nu$. The measure $\nu$ has a representation as an integral of the Hausdorff measure $m_{\lambda}$ if and only if $0<D^{\lambda} \nu(x)<\infty$ with probability 1 .

Let $x$ be chosen at random, distributed according to $\nu$. Consider the set $Z$ of all intervals $I\left(\gamma_{1}, \gamma_{2}, \ldots, \gamma_{n}\right)$ in $\Xi$ containing $x$. The set $Z$ is totally ordered by inclusion. Recall that for $k \leq n, I\left(\gamma_{1}, \gamma_{2}, \ldots, \gamma_{n}\right) \subset$ $I\left(\beta_{1}, \beta_{2}, \ldots, \beta_{k}\right)$ if and only if $\gamma_{i}=\beta_{i}$ for $1 \leq i \leq k$. Thus corresponding to $x$ there is an infinite sequence $\left(\gamma_{1}, \gamma_{2}, \ldots\right)$ of indices and $Z$ contains just those intervals of the form $I\left(\gamma_{1}, \gamma_{2}, \ldots, \gamma_{n}\right)$, the indices being the first 
$n$ terms of the sequence. This gives a one-to-one correspondence between the points of $F$ and infinite sequences $\left(\gamma_{1}, \gamma_{2}, \ldots\right)$ of indices. We note that the functions $g_{0}$ and $g_{1}$ are equivalent to right shifts on the set of sequences of indices.

Choosing the point $x$ at random is equivalent to choosing the sequence $\left(\gamma_{1}, \gamma_{2}, \ldots\right)$ at random. We describe a method for choosing the sequence which makes $\nu$ the distribution of $x$. Choose the $\gamma_{n}$ independently, each $\gamma_{n}$ equalling $s_{i}$ with probability $t_{i}, i=0,1$. We then have

$$
P\left(x \in I\left(\gamma_{1}, \gamma_{2}, \ldots, \gamma_{n}\right)\right)=t_{\gamma_{1}} t_{\gamma_{2}} \cdots t_{\gamma_{n}},
$$

as required.

We can now calculate $D^{\lambda} \nu(x)$ thusly:

$$
\begin{aligned}
D^{\lambda} \nu(x) & =\limsup _{n \rightarrow \infty} \frac{\nu I\left(\gamma_{1}, \gamma_{2}, \ldots, \gamma_{n}\right)}{\lambda\left(\operatorname{diam} I\left(\gamma_{1}, \gamma_{2}, \ldots, \gamma_{n}\right)\right)} \\
& =\limsup _{n \rightarrow \infty} \frac{t_{\gamma_{1}} \cdots t_{\gamma_{n}}}{\lambda\left(s_{\gamma_{1}} \cdots s_{\gamma_{n}}\right)} .
\end{aligned}
$$

We take the logarithm of both sides:

$$
\begin{aligned}
\log D^{\lambda} \nu(x) & =\limsup _{n \rightarrow \infty}\left[\sum_{i=1}^{n} \log t_{\gamma_{i}}-\Lambda\left(\sum_{i=1}^{n} \log s_{\gamma_{i}}\right)\right] \\
& =\limsup _{n \rightarrow \infty}\left(u_{n}-\Lambda\left(v_{n}\right)\right),
\end{aligned}
$$

where $\Lambda(u)=\log \lambda\left(e^{u}\right)$. Thus we can rephrase our problem in terms of a random walk on a lattice. In a plane with coordinates $u$ and $v$, with initial position $(0,0)$, we take randomly chosen steps. Each step is equal to the vector $(\Delta u=\log q, \Delta v=\log p)$ with probability $p$ and equal to the vector $(\Delta u=\log r, \Delta v=\log (1-p))$ with probability $(1-p)$. If $\left(u_{n}, v_{n}\right)$ is our position at the $n$th step, does there exist a function $\Lambda:(-\infty, a) \rightarrow$ $(-\infty, \infty)$ such that

$$
-\infty<\limsup _{n \rightarrow \infty}\left(v_{n}-\Lambda\left(u_{n}\right)\right)<\infty
$$

with probability 1 ?

First suppose we try a function of the form $\Lambda(u)=u d$, equivalent to setting $\lambda(t)=t^{d}$. Thus we consider

$$
\log D^{\lambda} \nu(x)=\limsup _{n \rightarrow \infty}\left(v_{n}-d u_{n}\right)=\limsup _{n \rightarrow \infty} \sum_{i=1}^{n}\left(\Delta v_{i}-d \Delta u_{i}\right) .
$$


Note $\Delta v_{i}-d \Delta u_{i}$ equals $(\log p-d \log q)$ with probability $p$ and equals $(\log (1-p)-d \log r)$ with probability $(1-p)$. The mean value of a step is therefore

$$
\alpha=p \log p+(1-p) \log (1-p)-d(p \log q+(1-p) \log r) .
$$

If $\alpha>0$ then this lim sup is $+\infty$ and $D^{\lambda} \nu(x)=\infty$ with probability 1 ; thus $\nu$ is singular with respect to $m_{\lambda}$ by Theorem 4.3. If $\alpha<0$ then this limsup is $-\infty$ and $D^{\lambda} \nu(x)=0$ with probability 1 ; thus $\nu$ is absolutely continuous with respect to $m_{\lambda}$ by Theorem 4.3. We therefore have $\operatorname{dim} \nu=d_{0}$, where

$$
d_{0}=\frac{p \log p+(1-p) \log (1-p)}{p \log q+(1-p) \log r}
$$

is the value of $d$ which makes $\alpha=0$. This is the formula dimension $=-$ entropy/Lyapunov exponent as in [12], p. 110. Also $\operatorname{dim} \mu=\operatorname{dim} \nu$ $+1=d_{0}+1$.

In the case $d=d_{0}$ we apply the following theorem from statistics:

4.4. LAW OF THE ITERATED LOGARITHM (Feller [3]). Let $\Delta a_{1}, \Delta a_{2}, \ldots$ be independent identically distributed random variables with mean $a_{0}$ and variance $\sigma^{2}$. Let

$$
a_{n}=\sum_{i=1}^{n} \Delta a_{i}
$$

Then with probability 1 ,

$$
\limsup _{n \rightarrow \infty} \frac{a_{n}-n a_{0}}{\sigma \sqrt{(2 n \log \log n)}}=1
$$

In the case at hand, we have $\Delta a_{i}=\Delta v_{i}-d_{0} \Delta u_{i}, a_{0}=0$, and $\sigma=0$ if $\log p \log r=\log (1-p) \log q ; \sigma>0$ otherwise. Thus in the case $\sigma>0$, we have

$$
\begin{aligned}
& \limsup _{n \rightarrow \infty} \frac{v_{n}-d_{0} u_{n}}{\sigma \sqrt{(2 n \log \log n)}}=1, \quad \text { and } \\
& \limsup _{n \rightarrow \infty}\left(v_{n}-d_{0} u_{n}\right)=\infty .
\end{aligned}
$$

Thus $D^{\lambda} \nu(x)=\infty$ with probability 1 and $\nu$ is singular with respect to $m_{\lambda}$ by Theorem 4.3. In the case $\sigma=0$, then $\Delta v_{n}-d_{0} \Delta u_{n}=0$ with probability 1 ; thus

$$
\log D^{\lambda} \nu(x)=\limsup _{n \rightarrow \infty}\left(v_{n}-d_{0} u_{n}\right)=0
$$


with probability 1 , and by Theorem $4.3, \nu$ has a representation as an integral of the Hausdorff measure $m_{\lambda}$, where $\lambda(t)=t^{d}$, and $d=d_{0}$ reduces to the value $\log p / \log q=\log (1-p) / \log r$.

So we have a special case where $\nu$ (and hence $\mu$ ) has a representation as an integral of Hausdorff measure. To treat the general case, we make use of an extended version of Theorem 4.4:

4.5. THEOREM (Feller [3]). Let $\Delta a_{1}, \Delta a_{2}, \ldots$ be independent identically distributed random variables with mean $a_{0}$ and variance $\sigma^{2}$, each taking only two possible values. Let

$$
a_{n}=\sum_{i=1}^{n} \Delta a_{i}
$$

Let $\phi:(0, \infty) \rightarrow(0, \infty)$. If the sum

$$
\sum_{n=1}^{\infty} \frac{\phi(n)}{n} \exp \left(-\frac{\phi^{2}(n)}{2}\right)
$$

converges, then with probability 1 ,

$$
a_{n}>n a_{0}+\sigma \sqrt{n} \phi(n)
$$

for only finitely many $n$. Conversely, if the sum (5) diverges, then with probability $1,(6)$ holds for infinitely many $n$.

First we treat the special case $q=r$, so that $\Delta u=\log q$ with probability 1 and $u_{n}=n \log q$. We also assume without loss of generality that $p<1 / 2$. We let $a_{n}=v_{n}$, so that $a_{0}=p \log p+(1-p) \log (1-p)$ and $\sigma=\sqrt{p(1-p)}(\log (1-p)-\log p)$.

For our purposes, the crucial point of Theorem 4.5 is that it describes two alternatives, each of which in appropriate circumstances occurs with probability 1 . Let $z$ be the random variable

$$
z=\limsup _{n \rightarrow \infty} v_{n}-\Lambda\left(u_{n}\right)-\Delta v_{1},
$$

so that $\log D^{\lambda} \nu(x)=z+\Delta v_{1}$. Suppose $z$ is finite with positive probability. We show that this contradicts Theorem 4.5. Let

$$
\varepsilon=(\log (1-p)-\log p) / 3 \text {. }
$$

Then there exists an interval $\left(z_{0}, z_{0}+\varepsilon\right)$ such that $z \in\left(z_{0}, z_{0}+\varepsilon\right)$ with positive probability $\delta$. Recall that $\Delta v_{1}$ equals $\log p$ with probability $p$ and equals $\log (1-p)$ with probability $(1-p)$. Thus the probability that $D^{\lambda} \nu(x) \in\left(z_{0}+\log p, z_{0}+\log p+\varepsilon\right)$ is at least $p \delta$ and the probability 
that $D^{\lambda} \nu(x) \in\left(z_{0}+\log (1-p), z_{0}+\log (1-p)+\varepsilon\right)$ is at least $(1-p) \delta$. Note that these two intervals are disjoint. Let

$$
z_{1}=z_{0}+(\varepsilon+\log p+\log (1-p)) / 2 .
$$

The probability that $D^{\lambda} \nu(x)<z_{1}$ is at least $p \delta$ and the probability that $D^{\lambda} \nu(x)>z_{1}$ is at least $(1-p) \delta$. Let

$$
\phi(n)=\left(\Lambda(n \log q)-n a_{0}+z_{1}\right) / \sigma \sqrt{n} .
$$

Then

$$
v_{n}>n a_{0}+\sigma \sqrt{n} \phi(n)
$$

holds for only finitely many $n$ with probability at least $p \delta$ and it holds for infinitely many $n$ with probability at least $(1-p) \delta$. This contradicts Theorem 4.5.

Hence the probability that $z$ and $\log D^{\lambda} \nu(x)$ are finite must be zero. Then, by Theorem 4.3, $\nu$ has no representation as an integral of Hausdorff measure $m_{\lambda}$. We state this result in a theorem.

4.6. THEOREM. Suppose that $\lambda$ is such that for each $a>0, \lambda(a t) / \lambda(t)$ is bounded as $t$ goes to 0 . If $q=r$ and $p \neq 1 / 2$, then the invariant measure $\mu$ generated by the modified baker's transformation (1) has no representation as an integral of Hausdorff measure.

We note that any Hausdorff measure on the square $S$ can be generated by an index function $\lambda$ such that $\lambda(a t) / \lambda(t)$ is bounded as $t$ goes to 0 , so that this hypothesis could be removed from the theorem.

For the more general case $q \neq r$, we introduce the variable

$$
w=u+v \frac{\log q-\log r}{\log (1-p)-\log p} .
$$

It is then true that

$$
w_{n}=n \frac{\log q \log (1-p)-\log r \log p}{\log (1-p)-\log p}=n \Delta w .
$$

We can then apply the techniques of this section to a random walk in the $(w, v)$ plane to show that $\nu$ has an integral representation if and only if

$$
\log p \log r=\log (1-p) \log q .
$$

The modified baker's transformation is one of the simplest possible dynamical systems. That a system as simple as this gives rise to a natural invariant measure with no representation as an indefinite integral of Hausdorff measure suggests that generically the natural invariant measure arising from a dynamical system will have no such representation. 
5. Classification of invariant measures by Hausdorff measures. We now consider the problem of comparing the degree of singularity of two invariant measures generated by different values of the parameters in the modified baker's transformation (1). Recall that we consider a finite measure $\mu$ more singular than a finite measure $\nu$ if there exists a Hausdorff measure $m_{\lambda}$ such that $\nu$ is absolutely continuous with respect to $m_{\lambda}$ while $\mu$ is singular with respect to $m_{\lambda}$. We first treat the restricted case $q=r$. Without loss of generality we assume $p<1 / 2$.

In the previous section we calculated the Hausdorff dimension of the measure $\nu$ on $[0,1]$ generated by the modified baker's transformation (1) to be:

$$
d_{0}=\frac{p \log p+(1-p) \log (1-p)}{\log q}
$$

We will consider index functions of the form

$$
\lambda(t)=t^{d_{0}} \exp (d \sqrt{\log (1 / t) \log \log \log (1 / t)}),
$$

corresponding to a function $\Lambda(u)=\log \lambda\left(e^{u}\right)$ of the form

$$
\Lambda(u)=d_{0} u+d \sqrt{-u \log \log (-u)} .
$$

Let us recall equation (4) from the previous section: with probability 1 ,

$$
\limsup _{n \rightarrow \infty} \frac{v_{n}-d_{0} u_{n}}{\sigma \sqrt{(2 n \log \log n)}}=1
$$

where $\sigma=\sqrt{p(1-p)}(\log (1-p)-\log p)$ is the standard deviation of $\Delta v_{i}-d_{0} \Delta u_{i}$ and $u_{n}=n \log q$. From equation (2), we have

$$
\begin{aligned}
\log D^{\lambda} \nu(x) & =\limsup _{n \rightarrow \infty} v_{n}-\Lambda\left(u_{n}\right) \\
& =\limsup _{n \rightarrow \infty} v_{n}-d_{0} u_{n}-d \sqrt{-u_{n} \log \log \left(-u_{n}\right)} \\
& =\limsup _{n \rightarrow \infty} v_{n}-d_{0} u_{n}-d \sqrt{-\log q} \sqrt{n \log \log n}
\end{aligned}
$$

Let

$$
d_{1}=\sqrt{\frac{-2}{\log q}} \sigma=(\log (1-p)-\log p) \sqrt{\frac{-2 p(1-p)}{\log q}} .
$$

Then when $d>d_{1}$, then (9) implies $\log D^{\lambda} \nu(x)=-\infty$ with probability 1 and $\nu$ is absolutely continuous with respect to $m_{\lambda}$ by Theorem 4.3. When $d<d_{1}$, then (9) implies $D^{\lambda} \nu(x)=\infty$ with probability 1 and $\nu$ is singular with respect to $m_{\lambda}$ by Theorem 4.3. 
Note the similarity between the determination of the appropriate value of $d_{1}$ and the definition of the Hausdorff dimension of a measure. With $\lambda$ as in (8), $\operatorname{dim} \nu$ is the critical value such that $\nu$ is absolutely continuous with respect to $m_{\lambda}$ when $d_{0}>\operatorname{dim} \nu$ and singular with respect to $m_{\lambda}$ when $d_{0}<\operatorname{dim} \nu$. When $d_{0}=\operatorname{dim} \nu$, we further have that $d_{1}$ is the critical value such that $\nu$ is absolutely continuous with respect to $m_{\lambda}$ when $d>d_{1}$ and $\nu$ is singular with respect to $m_{\lambda}$ when $d<d_{1}$. This criterion allows us to define the quantity $d_{1}$ for any measure, not just those arising from the modified baker's transformation (1). Moreover, for a set $E$, we can define a critical value $d_{1}$ such that $m_{\lambda} E=0$ when $d>d_{1}$ and $m_{\lambda} E=\infty$ when $d<d_{1}$. Besides classifying measures according to degree of singularity and sets according to size, $d_{1}$ can be used to bound the errors involved in estimates using the dimension. We suggest the name paradimension for the quantity $d_{1}$.

The usefulness of paradimension for classifying invariant measures in dynamical systems depends on its taking a spectrum of values for a family of measures, as opposed to being uniformly zero, for example. The analysis in this paper shows that this is true for the family of measures arising from the modified baker's transformation. Hausdorff measures with index functions of the form (8) appear also in a theorem by Makarov [5] concerning the following situation. Let the unit disc be mapped conformally onto the interior of a Jordan curve $\Gamma$ and let $\nu$ be the image under this mapping of Lebesgue measure on the boundary of the circle; thus $\nu$ is supported on $\Gamma$. Makarov's result, stated in our terminology, is that the dimension of $\nu$ is necessarily 1 while its paradimension may range from zero to some upper bound. Further work by Przytycki, Urbański, and Zdunik [6] ties this result in with the study of measures on repellers in dynamical systems and includes calculations of the paradimension of some such measures.

For an arbitrary ergodic invariant measure, the Hausdorff dimension can be obtained from the following formula for Hausdorff dimension at a point $x$ :

$$
\operatorname{dim} \nu=\liminf _{\varepsilon \rightarrow 0} \frac{\log \nu B(x, \varepsilon)}{\log \varepsilon},
$$

where $B(x, \varepsilon)$ denotes the ball of radius $\varepsilon$ centered at $x$. For an ergodic invariant measure in a dynamical system, the lim inf is independent of $x$ except on a set of $\nu$-measure zero. In a more general context, we must take the essential infimum over $x$. Proof of an essentially equivalent formula is given in Tricot [10]. This formula is often used in computations of the 
dimension as in [12]. We have found an analogous formula for the paradimension; the paradimension of a measure $\nu$ at a point $x$ is given by

$$
d_{1}(\nu)=\limsup _{\varepsilon \rightarrow 0} \frac{\log \nu B(x, \varepsilon)-\operatorname{dim} \nu \log \varepsilon}{\sqrt{(\log 1 / \varepsilon \log \log \log 1 / \varepsilon)}} .
$$

Again, for an invariant ergodic measure, the value of the lim sup is independent of $x$ except on a set of $\nu$-measure zero. The proof of this formula is rather lengthy, so we do not present it here.

One application of (10) is to calculate $d_{1}$ in the case $q \neq r$. Equation (4) still holds; we thus have with probability 1 :

$$
\limsup _{n \rightarrow \infty} \frac{v_{n}-d_{0} u_{n}}{\sqrt{(n \log \log n)}}=\sigma \sqrt{2}
$$

where $\sigma^{2}$ is the variance of the random variable $\Delta v_{i}-d_{0} \Delta u_{i}$; thus

$$
\sigma=\frac{-|\log p \log r-\log (1-p) \log q|}{p \log q+(1-p) \log r} \sqrt{p(1-p)} .
$$

We also have

$$
\lim _{n \rightarrow \infty} \frac{-u_{n} \log \log \left(-u_{n}\right)}{n \log \log n}=-p \log q-(1-p) \log r
$$

with probability 1 . We can thus calculate from (10):

$$
\begin{aligned}
d_{1}(\nu) & =\limsup _{n \rightarrow \infty} \frac{\log \nu B\left(x, e^{u_{n}} / 2\right)-d_{0} u_{n}}{\sqrt{\left(-u_{n} \log \log \left(-u_{n}\right)\right)}} \\
& =\limsup _{n \rightarrow \infty} \frac{v_{n}-d_{0} u_{n}}{\sqrt{\left(-u_{n} \log \log \left(-u_{n}\right)\right)}} \\
& =\frac{\sigma \sqrt{2}}{\sqrt{(-p \log q-(1-p) \log r)}} \\
& =\frac{|\log p \log r-\log (1-p) \log q| \sqrt{2 p(1-p)}}{(-p \log q-(1-p) \log r)^{3 / 2}} .
\end{aligned}
$$

In going from the first to the second step above we have replaced $\log \nu B\left(x, e^{u_{n}} / 2\right)$ by $v_{n}$, which is not quite the same thing, but this restriction to intervals in the collection $\Xi$ does not affect the value of the lim sup. 
6. Acknowledgments. This research was supported in part by a grant from the Naval Academy Research Council.

I am indebted to the referees for their conscientious work. The present form of this article owes much to their suggestions. Their advice was particularly helpful in the revision of $\S \S 3$ and 5 .

\section{REFERENCES}

[1] M. Benedicks and L. Carleson, On iterations of $1-a x^{2}$ on $(-1,1)$, Ann. of Math., (2) 122 (1985), 1-25.

[2] P. Collet and J.-P. Eckmann, Iterated Maps on the Interval as Dynamical Systems, Birkhäuser, Boston 1980.

[3] W. Feller, An Introduction to Probability Theory and Its Applications, Volume One, John Wiley \& Sons, Inc., New York 1950.

[4] M. V. Jakobson, Absolutely continuous invariant measures for one-parameter families of one-dimensional maps, Commun. Math. Phys., 81 (1981), 39-88.

[5] N. G. Makarov, On the distortion of boundary sets under conformal mappings, Proc. London Math. Soc. (3), 51 (1985), 369-384.

[6] F. Przytytcki, M. Urbański, and A. Zdunik, Harmonic, Gibbs, and Hausdorff measures on repellers for real analytic maps, (preprint).

[7] C. A. Rogers, Hausdorff Measures, Cambridge University Press: Cambridge 1970.

[8] C. A. Rogers and S. J. Taylor, Additive set functions in Euclidean space II, Acta Math., 109 (1963), 207-240.

[9] D. Ruelle, Bowen's formula for the Hausdorff dimension of self-similar sets. In: Scaling and self-similarity in physics: renormalization in statistical mechanics and dynamics, ed. Jürg Fröhlich, Birkhäuser, Boston 1983.

[10] C. Tricot, Jr., Two definitions of fractional dimension, Math. Proc. Camb. Phil. Soc., 91 (1982), 57-74.

[11] W. D. Withers, Folding polynomials and their dynamics, Amer. Math. Monthly, (to appear).

[12] L.-S. Young, Dimension, entropy, and Lyapunov exponents, Ergod. Th. \& Dynam. Sys., 2 (1982), 109-124.

Received April 8, 1986 and in revised form October 13, 1986.

U. S. Naval ACademY

ANNAPOLIS, MD 


\title{
PACIFIC JOURNAL OF MATHEMATICS \\ EDITORS
}

\author{
V. S. VARADARAJAN \\ (Managing Editor) \\ University of California \\ Los Angeles, CA 90024 \\ HERBERT Clemens \\ University of Utah \\ Salt Lake City, UT 84112 \\ R. FINN \\ Stanford University \\ Stanford, CA 94305
}

HERMANN FLASCHKA

University of Arizona

Tucson, AZ 85721

RAMESh A. GANGOLLI

University of Washington

Seattle, WA 98195

VAUGHAN F. R. JONES

University of California

Berkeley, CA 94720
ROBION KIRBY

University of California

Berkeley, CA 94720

C. C. MOORE

University of California

Berkeley, CA 94720

HAROLD STARK

University of California, San Diego

La Jolla, CA 92093

\section{ASSOCIATE EDITORS}

\author{
R. ARENS \\ E. F. BECKENBACH \\ B. H. NEUMANN \\ F. WOLF \\ K. YOSHIDA \\ (1906-1982)

\section{SUPPORTING INSTITUTIONS} \\ UNIVERSITY OF ARIZONA \\ UNIVERSITY OF BRITISH COLUMBIA \\ CALIFORNIA INSTITUTE OF TECHNOLOGY \\ UNIVERSITY OF CALIFORNIA \\ MONTANA STATE UNIVERSITY \\ UNIVERSITY OF NEVADA, RENO \\ NEW MEXICO STATE UNIVERSITY \\ OREGON STATE UNIVERSITY \\ UNIVERSITY OF OREGON \\ UNIVERSITY OF SOUTHERN CALIFORNIA \\ STANFORD UNIVERSITY \\ UNIVERSITY OF HAWAII \\ UNIVERSITY OF TOKYO \\ UNIVERSITY OF UTAH \\ WASHINGTON STATE UNIVERSITY \\ UNIVERSITY OF WASHINGTON
}

The Supporting Institutions listed above contribute to the cost of publication of this Journal, but they are not owners or publishers and have no responsibility for its content or policies.

Mathematical papers intended for publication in the Pacific Journal of Mathematics should be in typed form or offset-reproduced (not dittoed), double spaced with large margins. Please do not use built up fractions in the text of the manuscript. However, you may use them in the displayed equations. Underline Greek letters in red, German in green, and script in blue. The first paragraph must be capable of being used separately as a synopsis of the entire paper. In particular it should contain no bibliographic references. Please propose a heading for the odd numbered pages of less than 35 characters. Manuscripts, in triplicate, may be sent to any one of the editors. Please classify according to the scheme of Math. Reviews, Index to Vol. 39. Supply name and address of author to whom proofs should be sent. All other communications should be addressed to the managing editor, or Elaine Barth, University of California, Los Angeles, California 90024.

There are page-charges associated with articles appearing in the Pacific Journal of Mathematics. These charges are expected to be paid by the author's University, Government Agency or Company. If the author or authors do not have access to such Institutional support these charges are waived. Single authors will receive 50 free reprints; joint authors will receive a total of 100 free reprints. Additional copies may be obtained at cost in multiples of 50 .

The Pacific Journal of Mathematics is issued monthly as of January 1966. Regular subscription rate: $\$ 190.00$ a year (5 Vols., 10 issues). Special rate: $\$ 95.00$ a year to individual members of supporting institutions.

Subscriptions, orders for numbers issued in the last three calendar years, and changes of address should be sent to Pacific Journal of Mathematics, P.O. Box 969, Carmel Valley, CA 93924, U.S.A. Old back numbers obtainable from Kraus Periodicals Co., Route 100, Millwood, NY 10546.

The Pacific Journal of Mathematics at P.O. Box 969, Carmel Valley, CA 93924 (ISSN 0030-8730) publishes 5 volumes per year. Application to mail at Second-class postage rates is pending at Carmel Valley, California, and additional mailing offices. Postmaster: send address changes to Pacific Journal of Mathematics, P.O. Box 969, Carmel Valley, CA 93924.

PUBLISHED BY PACIFIC JOURNAL OF MATHEMATICS, A NON-PROFIT CORPORATION Copyright (C) 1987 by Pacific Journal of Mathematics 


\section{Pacific Journal of Mathematics}

Vol. 129, No. $2 \quad$ June, 1987

Pere Ara, Matrix rings over $*$-regular rings and pseudo-rank functions . . . . 209

Lindsay Nathan Childs, Representing classes in the Brauer group of quadratic number rings as smash products $\ldots \ldots \ldots \ldots \ldots \ldots \ldots \ldots 243$

Dicesar Lass Fernandez, Vector-valued singular integral operators on $L^{p}$-spaces with mixed norms and applications $\ldots \ldots \ldots \ldots \ldots \ldots \ldots \ldots \ldots \ldots \ldots$

Louis M. Friedler, Harold W. Martin and Scott Warner Williams,

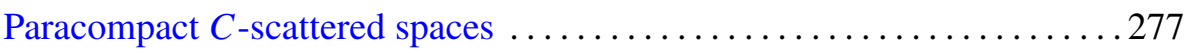

Daciberg Lima Gonçalves, Fixed points of $S^{1}$-fibrations . . . . . . . . . . 297

Adolf J. Hildebrand, The divisor function at consecutive integers . . . . . . 307

George Alan Jennings, Lines having contact four with a projective

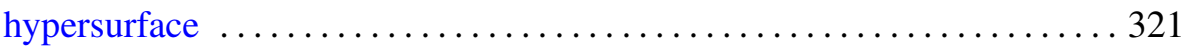

Tze-Beng Ng, 4-fields on $(4 k+2)$-dimensional manifolds $\ldots \ldots \ldots \ldots . \ldots 337$

Mei-Chi Shaw, Eigenfunctions of the nonlinear equation $\Delta u+v f(x, u)=0$ in $R^{2}$

Roman Svirsky, Maximally resonant potentials subject to $p$-norm constraints

Lowell G. Sweet and James A. MacDougall, Four-dimensional

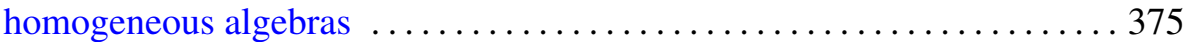

William Douglas Withers, Analysis of invariant measures in dynamical systems by Hausdorff measure 